removed by litholapaxy. The bladder was washed out daily, with the result that the urine became acid, but there was no diminution in the pus or frequency.

On December 16th Mr. Bickersteth, of Liverpool, and others being present, I removed the prostate. There was considerable difficulty in opening the bladder suprapubically. owing to the scar tissues being matted together, and still greater difficulty in enucleating the prostate, owing to the inflammatory adhesions, resulting from the previous operation, between the inner margins of the prostate and the walls of the bladder, though the gland came away readily from the triangular ligament. A portion of the prostatic urethra came away adherent to the gland. Uninterrupted recovery. The wound was closed lecember 29 th, thirteen days after the operation. The patient is now quite well, walking about the wards, able to retain and pass his urine as well as he ever did.

The prostate (Fig. 4, p. 1186 ), which weighs $x_{4}^{\prime} \mathrm{oz}$., is jagged along the posterior aspect of the right lobe, where it was adherent to and matted with the bladder wall. This is the third case in which my operation of complete enucleation of the prostate has been entirely successiul after McGill's operation had failed. The previous operator in this case has persistently in his writings and otherwise minimized and misrepresented the nature and scope of my operation, as being identical with McGill's. It is the irony of fate that this case, in which Mecill's operation performed by him had entirely failed to bring relief to the patient, should have fallen into my hands to be completely cured by total extirpation of the prostate.

$$
\text { CAse LXXirr. }
$$

A gentleman, aged 76 , sent by Dr. T. D. Griffiths, Swansea, seen with Dr. Homerton, London, December $x_{5}$ th, 1903 . Prostatic symptoms for three years : retention ten months ago; catheter employed since then with much pain in passing it; great irritability of the bladder; cannot sleep from the frequency of mieturition; urine acid but contains much pus and mucus; prostate greatly enlarged, smooth, soft, movable, felt bimanually ; patient's health much impaired.

On December rgth, Mr. Thomson Walker and Dr. Homerton assisting, I removed the prostate, weighing $4 \frac{3}{4} 0 \%$, as usual. There was very little bleeding or shock. The patient has had no bad symptom, and is now convalescent.

Note.-During the period that has elapsed since this lecture was delivered on January 6th, 1904, I have performed this operation in a be submitted for the consideration of the prolession.

REFERENCES.

1 July 2oth, rgor. 2 February rst, July 26th. and November 8 th, 1902 April i8th, July 4 th, and October 17 th, 1903 . 3 BRITISH MEDICAL JOURNAL, October 17 th, 1903.

\section{TOTAL PROSTATECTOMY}

DEDUCTIONS TO BE DRAWN FROM THE PRESENCE OF STRIATED MUSGLE FIBRE IN THE "GAPSULE" OF THE PARTS REMOVED.

BY CUTHBERT S. WAL:ACE, B.S., F.R.C.S., Assistant Surgeon to St. Thomas's Hospital and the East London Hospital for Children.

Mr. Thomson WALKer, in a recent communication read before the Royal Medical and Chirurgical Society, ${ }^{\prime}$ has drawn attention to the presence of striped muscle fibre on the anterior aspect of the prostate. He describes it as surrounding the apex of the organ and then as passing up on the anterior surface of the gland between the "capsule" and the posterior layers of the recto-vesical sheath of the prostate. (The prostatic plexus of veins lies between the layers of the recto-vesical fascia; consequently there will be a layer of the recto-vesical sheath between the veins and the layer of striped muscle.) In another part of the same paper, when describing the "capsule" on a specimen removed by prostatectomy, it was stated that striped muscle fibre was found in the anterior part of the "capsule." From this it was argued that the line of cleavage in the process of enucleation must have passed outside the confines of the gland, and that therefore the prostatectomy was complete.

In microscopic sections of the normal prostate made at right angles to the long axis of the urethra and midway between the extremities of the gland, the following features in the relation of this striated muscle may be noted.

At the most anterior part of such a transverse section the striated muscle fibres take a transverse or horizontal direciion. The bundles are well marked and are separated from each other by a little fine areolar tissue. Near the urethra the bundles become more widely separated, and between them are seen to lie well-marked fibrous tissue and unstriped muscle fibres. Traced outwards towards the lateral parts of the prostate, the striped muscle fibres lose themselves in the substance of the organ. Passing inwards towards the urethra the striped muscle fibres become more and more widely separated by the fibro-muscular stroma of the gland until at last the latter predominates, the striped fibres becoming fewer and fewer until they cease altogether. Some time, however, before this happens the gland tissue makes its appearance, so that striped muscle fibre, fibro-muscular stroma, and gland tissue are found in the same field; and it is possible to see gland tissue with striped muscle fibres lying on both its central and peripheral aspect, so intimately is the striped fibre insinuated into the peripheral portion of the gland.

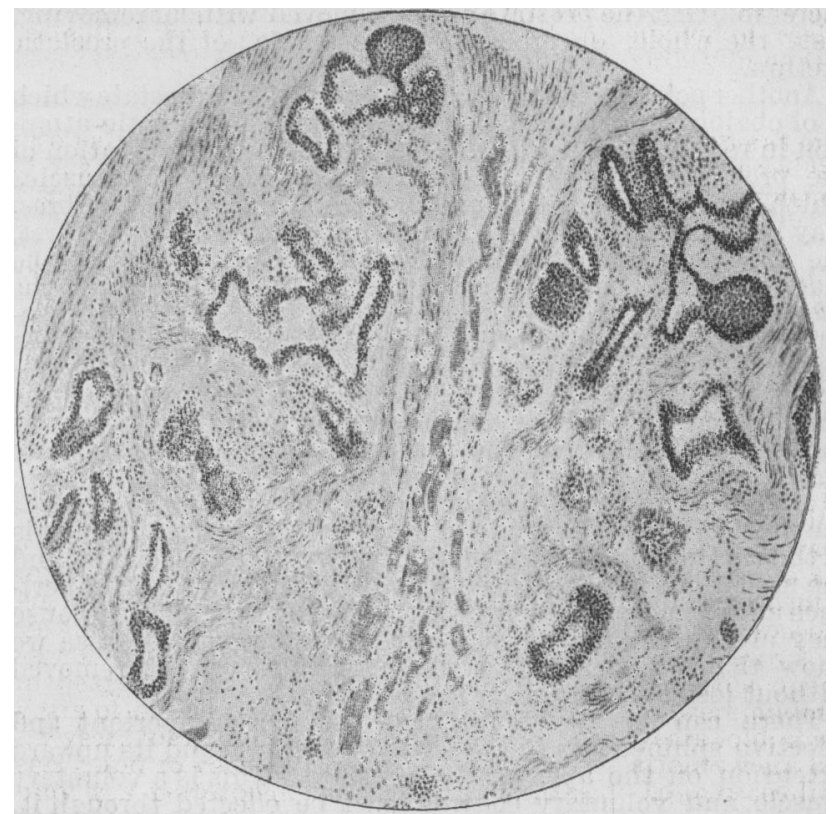

Fig. 1.-Showing a portion of the periphery of the prostate of a young adult. $\Lambda$ group of striated muscle fibres is seen penetrating the stroma between the gland acini.

It thus foliows that the presence of striped muscle fibre in the "capsule" of an enurleated prostate is not to be taken as an irrefragable proof that the line of enucleation has passed outside the confines of the gland, and that therefore the prostatectomy is complete. The completeness, as in other cases, can only be established by a microscopic demonstration of the absence of gland tissue in the parts left behind.

REFERENCE.

1 BRITISH Medical JouRnal, March 26th, 1904, p. 728.

\section{THE RELATIVE ADVANTAGES OF SUPRAPUBIC aNd PERINeal PRostatectomy.*}

By EDWARD DEANESLY, B.Sc.LoNI., F.R.C.S.EN(i., Honorary Surgeon, Wolverhampton and Staffordshire General Hospital.

THE prostate must be regarded both anatomically and functionally as an accessory portion of the urethra, to which it is inseparably attached. Its relation to the bladder is much less intimate. The normal prostate is entirely extravesical, and its internal projection within the bladder is hardly visible when the bladder and urethra are slit open from the front. Seen in their normal relations in the living body by means of the cystoscope, the difference between the male and female bladders is much less than would be expected, and all that is seen of the prostate is a low median-grooved projection at the posterior part of the orifice of the urethra. The median groove corresponds to the anterior aspect of the projecting uvula vesicae, and in some cases the uvula itself is seen as a small round projection in the middle of the groove.

The prostatic urethra, $\mathrm{I}_{4}^{1}$ in. in length, is completely embraced by the prostate, but a much thicker portion of prostate covers it behind than in front. Moreover, the anterior portion consists mainly of unstriped muscular fibres, and in some cases no gland tissue at all exists in front of the urethra. The prostatic utricle and seminal ducts open into the projecting veru montanum near the centre of the prostatic urethra, and above the centre are the openings of the prostatic ducts. The connexion of the urethra with the surrounding prostatic

* Read beiore the Staffordshire Branch of the British Medical Association. 
tissue is so intimate that it is impossible with the sharpest instruments by any ordinary means of dissection to separate the normal urethra from the prostate. When the prostate is enlarged the urethra participates in the process, and becomes distorted and elongated, sometimes to the extent of more than double its original length. To any one who examines an anatomical specimen showing this condition it is simply incredible that the prostate can be removed without removing, also, the whole, or practically the whole, of the prostatic urethra.

Another point in the surgical anatomy of the prostate which is of obvious importance, but which has received little attention in recent discussions on prostatectomy, is the relation of the prostate to the sphincters of the bladder. The muscles which, from their position and the arrangement of their fibres, may be presumed to have a sphincter action are three. First, the thicker portion of the circular muscular coat of the bladder which surrounds the neck and the orifice; next, the intrinsic muscle fibres of the prostate; and, lastly, the constrictor urethrae and a layer of circular fibres which extends upwards from it, and embraces the apex of the prostate. The first two of these are unstriped (involuntary) muscles; the last consists of ordinary striated muscular fibres. It was thought by Henle that the muscular tissue of the prostate formed the effective sphincter of the bladder; but although, as already mentioned, the portion of the prostate in front of the urethra consists mainly of muscle fibres crossing the axis of the urethra, and others similarly disposed are seen behind the urethra in transverse sections of the gland, their importance as sphincters cannot be great, in the first place because they are absent in women, and, in the second, because we know that practically the whole prostate can be removed without loss of control over the bladder.

There can be no doubt that the most important and effective sphincter is the constrictor urethrae and its upward extension on the apex of the prostate. This is a voluntary muscle and voluntary control must be effected through it. just as voluntary control of the anus depends on the external sphincter ani. In both cases of course there exists also an internal or involuntary sphincter muscle composed of a strengthened band of the normal circular muscular coat of the viscus. When a catheter is introduced within any part of the prostatic urethra no urine flows unless the patient strains - that is, unless the bladder contracts or is compressed by the abdominal walls. This shows that the prostatic urethra is normally closed, and is not, in fact, a portion of the bladder. The force closing it, however, presumably the contraction of the circular fibres of the neck of the bladder, is very small. for once the point of the catheter is in the prostatic urethra very little hydrostatic pressure suffices to overcome it and to allow fluid to enter the bladder. On the other hand, to inject fluid through the anterior urethra and overcome the resistance of the constrictor urethrae a much higher pressure is required.

The relation of the prostate to surrounding structures, especially to the pelvic fascia, has lately been the subject of much controversy, and is of great surgical importance. This controversy arose out of the well-known operations of Mr. P. J. Freyer, to whom, whatever view we may take of his anatomical contentions, is due the credit of having demonstrated that complete prostatectomy from a surgical point of view is not only possible but much simpler than had previously been supposed. Mr. Freyer's contentions were, first, that the enlarged prostate had a definite capsule of its own from which it could be enucleated with the fins ar; and, secondly, that in some cases, at any rate, it was possible to remove the prostate in one or more pieces, leaving an intact urethra behind.

I think most of those who have followed the controversy are now agreed that neither of those contentions has been sustained. The observations of Mr. S. G. Shattock and of Mr. Cuthbert S. Wallace seem to me absolutely conclusive on the main points. There only remains, in order to clear up the confusion, that surgeons should agree upon a common nomenclature of the structures involved. All are agreed that after Freyer's operation some kind of prostatic capsule is left behind. Whether this is a true fibrous capsule or a condensed portion of the prostatic cortex compressed by the adenomatous growths within is immaterial from a surgical point of view. It is certain that the normal prostate has no such capsule, and the investments of the enlarged adenomatous prostate may be described best as consisting of an outer fibrous sheath formed by the'recto-vesical fascia, and an inner capsule or cortex derived from the gland itself. Between these two investments lie the prostatic plexus of veins, which are not injured by the operation of enucleation.

It is obvious that the prostate may be approached surgically by two routes: (1) through the bladder, and (2) from without the bladder, through the rectum or perineum. The main object of this paper is to discuss the relative advantages of these two routes. At the present time, owing to the brilliant series of operations published by Mr. Freyer, the suprapubic route is that most generally adopted. Suprapubic cystotomy is a simple and familiar operation, and gives ready access to the prostate from within the bladder. The mucous membrane over the prostate is incised over one or both sides of the prostate, and the latter is then easily enucleated by the finger, aided by the fingers of the other hand in the rectum. I have performed this operation four times, and in three out of the four the prostate was shelled-out completely without difficulty and with little haemorrhage. 'In the fourth it was simply impossible to enucleate the prostate, which was of the hard fibrous variety, and the operation had to be completed by the perineal route. The recto-vesical sheath had, however, been opened, and the patient succumbed to pelvic cellulitis at the end of a fortnight. In this case there was nothing to distinguish the fibrous from the adenomatous variety of prostatic enlargement, either by rectal palpation or by cystoscopic examination. It was considerably enlarged, and projected within the bladder like a cervix uteri, just in the same manner as in the other three cases in which enucleation was easy. Had this case been operated on by the perineal method without the previous injury inflicted by the unsuccessful efforts from above, the whole or a sufficient amount of the prostate could have been safely removed under the guidance of direct vision. Or, if this had proved impossible, the operation could have been abandoned without inflicting any serious injury.

Another objection to the suprapubic route is the great depth of the prostate from the surface in stout subjects. Owing to this cause considerable laceration of the prevesieal cellular tissue is difficult to avoid, and exposes the patient to two serious dangers-prolonged oozing of blood into the subperitoneal tissues and subsequent pelvic cellulitis. This occurred in one of my cases, and although the prostate was easily enucleated in two large smooth masses, death occurred on the third day.

Iastly, in the suprapubic operation the subsequent drainage of the bladder is effected against gravity, and is often a source of serious trouble. The pouch left in the bottom of the bladder is never completely emptied, the urine generally becomes ammoniacal, and the wound often becomes sloughy and coated with phosphates. Owing to these causes healing of the wound may be delayed for many weeks.

These objections to the suprapubic incision are so obvious that the alternative perineal route has been advocated and practised by many surgeons both before and since Freyer's cases were published. Mr. J. H. Nicoll, of Glasgow, in 1894 advocated a preliminary suprapubic cystotomy followed by a median perineal incision. The prostate was then pushed into the perineal wound by two fingers in the bladder from above, and a sufficiency of redundant prostatic tissue clipped away with scissors without opening the urethra or bladder. Bruce Clarke in 1900 advocated a very similar procedure, carried out in two stages. This operation has certain obvious advantages, especially in such a case as that I have already mentioned, where the prostate is so densely fibrous that enucleation is impossible.

A much superior perineal method, which I shall now describe, has been elaborated by M. Robert Proust, of Paris, and is described in detail in an admirably illustrated monograph published in 1903. After verifying the steps of the operation on the cadaver, I have employed this method on a man whom I show to-day and whose case I will first briefly relate.

He is now 65 years of age. In September, rgor, the late Mr. Vincent Jackson removed a large stone by suprapubic cystotomy, and at the same time snipped away some small portions of the prostate which projected within the bladder. As a result of this operation he was much benefited, and was able to dispense with the catheter, on which he had for some time previously been entirely dependent. Symptoms of inger an ineffectur stone recurred, and in calculi lying in a narrow-mouthed pouch at the base of the bladder, calculi lying in a narrow-mouthed pouch at the base of the bladder, uteri into the bladder. After this operation he remained well for twelve months ; another stone was then found, and I advised prostatectomy. Influenced by the considerations already mentioned, and also by the presence of a considerable ventral hernia, the result of the 
two previous suprapubic operations, I selected the perineal route, and carried it out by Proust's method on February 18 th, 1904. The operation proved rapid and simple in performance, and satisfactory in its result. Convalescence was rather slow, but unattended by any special difficulties, and the patient is now-ten weeks after the operation-in good health. The urine is acid and clear, and micturition forcible and free from pain. There remains, however, some weakness of the sphincter of the bladder, and he is unable to retain urine berond two sphincte
liours.

M. Proust, in his work, makes no reference to Freyer's procedure; but I may point out that Freyer's discovery (for without disparaging previous operators it really amounts to a discovery), namely, that in the majority of cases of enlarged prostate the whole mass can be easily enucleated, is of equal value, whether the prostate is approached from above or from below. The final stage of the operation-the enucleation-is the same in both cases, except'that by M. Proust's procedure the prostate is not only completely exposed to sight and touch but rendered quite superficial, so that the whole operation can be carried out with precision and deliberation. The steps by which this is accomplished are as follows, and great importance is attached to the precise performance of each step before proceding to the next. These steps are illustrated by enlarged copies of Proust's illustrations here shown.

In the first place the patient is placed in what is termed the inverse perineal position-that is, the lithotomy positionwith the pelvis raised by a special table or by blocks to such a height that the surface of the perineum is almost horizontal. A catheter is passed and left in the bladder. A curved incision with its convexity forwards is then made from one ischial tuberosity to the other, the summit of the curve meeting the middle line two fingerbreadths in front of the anus. The skin and fat are dissected back, and the bulbocavernosus and ischio-cavernosus are defined. A small muscular bundle, which Proust terms the superficial anobulbar raphe, passing from the hinder end of the bulb to the anus, is put on the stretch and divided transversely. The anterior end of this band being drawn upwards and forwards the bulb is lifted with it, and two important landmarks are row exposed and defined-the anterior edges of the levator ani on each side and in the middle line between them a band of muscular fibres uniting the membranous arethra to the rectum, called by Proust the rectourethral muscle. The recognition of this structure is the most important step in the operation because it is the key to the recto-vesical space. It is divided transversely, keeping the scissors parallel to the membranous urethra so as to avoid wounding the latter. When this cut is made accurately it at once opens the retro-vesical space, and the orostate and bladder are easily separated from the rectum as higli as the reflection of peritoneum by means of the two forefingers. If a suitable retractor is now inserted and the rectum and anus drawn strongly backwards, the posterior surface of the prostate is not only exposed to view but is dragged down into a quite superficial position, in which it can be easily handled. In order to make it project still more the prostatic urethra is opened at its apex and a sharplycurved sound passed into the bladder. If the beak of the instrument is now turned round so that it looks towards the operator, the prostate can be drawn down and steadied in any desired position.

It is now easy to open the prostatic capsule and by mean of the finger and blunt elevators to enucleate completely the prostate on its peripheral aspect, leaving it attached to the bladder merely by the prostatic urethra. The latter is next incised along its floor throughout its whole length but without trespassing on the neck of the bladder. Inserting the finger inside the open urethra and using sharp scissors, each lateral half of the prostate, according to Proust, may be cut away from the urethra without wounding the latter, and the manner of doing so is clearly shown in one of his figures. By the use of sharp instruments and by making the incisions in the substance of the prostatic tissue itself, it is of course theoretically possible to remove the bulk of the prostate, and leave the prostatic urothra as a completely isolated tube attached to the membranous urethra at one end and to the neck of the urethra at the other. The mere description of the result of this procedure makes it difficult to believe that this is really possible, except. perhaps. in cases where the enlarged prostate projects but slightly within the bladder. Where it projects into the bladder, as it does in the great majority of cases, to the extent of one-third or even one-half of its bulk. I do not believe it is possible to remove the whole organ without taking away the greater part, if not the whole, of the prostatic urethra. Without, however, accepting the possibility of preserving the prostatic urethra as a normal procedure, it is one of the principal merits of the perineal method, as opposed to the suprapubic, that the whole operation is conducted with a full and clear view of the field of operation. It is for this reason much more adaptable to the varied conditions of particular cases. It is by no means certain that complete removal of the whole of the enlarged prostate is required in all cases, or is, indeed, possible in every case. Where, therefore, enucleation is found to offer unusual difficulty, such as that due to the densely fibrous form of prostatic enlargement, it would be easy by the perineal method to clip away with scissors the bulk of the gland without opening the bladder or the urethra, except by means of the median incision already described. This incision, however, should never be omitted, for the amount and character of the internal projection, even if previously determined by the cystoscope, as is always advisable, must be confirmed by a finger in the bladder. If by this means it is found that the removal of the portions of gland from outside the bladder has not sufficiently lessened the projection within it, or especially if any pedunculated projecting mass is felt, the projecting portions can be easily reduced or removed by suitable gouge forceps passed into the bladder.

The after-treatment is simple. The breach in the urethra or bladder is partly closed by sutures not passing through the mucous membrane, and a large rubber tube is brought out through the centre of the perineum; the rest of the perineal wound is sutured. The bladder is irrigated daily, the tube being removed as soon as the state of the urine and wound permits. The resulting perineal fistula then rapidly closes, and normal micturition is resumed.

In conclusion, I may briefly summarize the principal advantages which prostatectomy by the perineal method appears to me to possess compared with the more familiar suprapubic method. It is anatomically more direct; it does not inflict a double wound in the bladder, and does not open the prevesical cellular tissue. Again, the whole operation can be performed deliberately, and under the direct guidance of sight as well as touch ; it permits, therefore, the operator to remove as much or as little of the prostatic overgrowth as the circumstances of the case or the nature of the enlargement render desirable or feasible. Lastly, the drainage of the bladder and subsequent wound treatment are much facilitated by the dependent opening. The conspicuous merit of Proust's operation compared with previous nerineal methods is the accuracy and certainty with which each step of the operation is performed, and the admirable access to sight and touch which it gives to the prostate and base of the bladder. When the steps of the operation are properly carried out, the exposure of the prostate is extremely rapid and simple, and the whole operation occupies no more time than the suprapubic operation. Perhaps the greatest merit of the operation is that it permits a precise examination of the whole prostate both within and without the bladder before the question of removal of the organ is finally decided. The preliminary stages of the operation, indeed, offer a method of exploring the bladder which for many purposes is much superior to suprapubic cystotomy. In those common cases, for example, where stone and enlarged prostate coexist, and where lithotrity is either difficult or inadvisable, owing to the liability of recurrence, it is usual to remove the stone by suprapubic cystotomy. Of late years the prostate has often been removed at the same time. Most surgeons are aware that suprapubic lithotomy in adults, whether accompanied or not by prostatectomy, is attended with a considerable mortality, but the difficulties imposed by the enlargement of the prostate and the depth of the bladder have hitherto made lateral or median perineal lithotrity even more difficult and dangerous. The latter difficulties have been entirely overcome by Proust's method, by which the bladder and prostate can be easily and safely opened from the perineum, either for exploration, for removal of calculi, or for removal of the whole or portions of the prostate.

Presentation.-Dr. W. Hooper Masters, on leaving Parkstone to take up his residence in Barnes, was presented with a walnut wood writing table, with desk and revolving chair, by the members of the Poole and Parkstone Natural History Society, of which he was president; he also received a silver matchbox and gold mounted cigarette holder from the Gardeners' Association. 